Published in final edited form as:

Clin Drug Investig. 2019 May ; 39(5): 441-451. doi:10.1007/s40261-019-00764-x.

\title{
Effect of CYP3A Inhibition and Induction on the Pharmacokinetics of Suvorexant: Two Phase I, Open-Label, Fixed-Sequence Trials in Healthy Subjects
}

\author{
Rebecca E. Wrishko ${ }^{1}$, Jacqueline B. McCrea ${ }^{1}$, Ka Lai Yee ${ }^{1}$, Wen Liu ${ }^{1}$, Deborah \\ Panebianco' ${ }^{1}$, Eric Mangin ${ }^{1}$, Manu Chakravarthy ${ }^{1}$, Maria P. Martinez-Cantarin ${ }^{2}$, Walter K. \\ Kraft $^{2}$ \\ ${ }^{1}$ Merck \& Co., Inc., Kenilworth, NJ, USA \\ ${ }^{2}$ Department of Pharmacology and Experimental Therapeutics, Thomas Jefferson University, \\ Philadelphia, PA, USA
}

\begin{abstract}
Background and Objectives-Suvorexant is an orexin receptor antagonist indicated for the treatment of insomnia, characterized by difficulties with sleep onset and/or sleep maintenance. As suvorexant is metabolized primarily by Cytochrome P450 3A (CYP3A), and its pharmacokinetics may be affected by CYP3A modulators, the effects of CYP3A inhibitors (ketoconazole or diltiazem) or an inducer (rifampin [rifampicin]) on the pharmacokinetics, safety, and tolerability of suvorexant were investigated.
\end{abstract}

\begin{abstract}
Methods-In two Phase I, open-label, fixed-sequence trials (Studies P008 and P038), healthy subjects received a single oral dose of suvorexant followed by co-administration with multiple once-daily doses of strong/moderate CYP3A inhibitors (ketoconazole/diltiazem) or a strong CYP3A inducer (rifampin). Treatments were administered in the morning: suvorexant $4 \mathrm{mg}$ with ketoconazole $400 \mathrm{mg}$ (Study P008; $N=10$ ), suvorexant $20 \mathrm{mg}$ with diltiazem $240 \mathrm{mg}$ (Study P038; $N=20$ ), and suvorexant $40 \mathrm{mg}$ with rifampin $600 \mathrm{mg}$ (Study P038; $N=10$ ). Area under the plasma concentration-time curve from time zero to infinity $\left(\mathrm{AUC}_{0-\infty}\right)$, maximum plasma concentration $\left(C_{\max }\right)$, half-life $\left(t_{1 / 2}\right)$, and time to $C_{\max }\left(t_{\max }\right)$ were derived from plasma concentrations of suvorexant collected at prespecified time points up to 10 days following CYP3A inhibitor/inducer co-administration. Adverse events (AEs) were recorded.
\end{abstract}

Jacqueline B. McCrea, jackie_mccrea@merck.com.

Data availability Merck Sharp \& Dohme Corp., a subsidiary of Merck \& Co., Inc., Kenilworth, NJ, USA's data sharing policy, including restrictions, is available at http://engagezone.msd.com/ds_documentation.php. Requests for access to the clinical study data can be submitted through the EngageZone site or via email to dataaccess@ merck.com.

Conflict of interest REW, JBM, KLY, WL, DP, EM, and MC are current or former employees of Merck Sharp \& Dohme Corp., a subsidiary of Merck \& Co., Inc., Kenilworth, NJ, USA, and may own stock and/or stock options. MPM-C was supported by National Institutes of Health Postdoctoral Training Grant No. T32GM008562. WKK has no disclosure to make.

Ethical approval All procedures performed in studies involving human subjects were in accordance with the ethical standards of the institutional and/or national research committee and with the 1964 Helsinki declaration and its later amendments or comparable ethical standards. This article does not contain any studies with animals performed by any of the authors.

Informed consent Informed consent was obtained from all individual subjects included in the study. 
Results-Co-administration with ketoconazole resulted in increased exposure to suvorexant [AUC ${ }_{0-\infty}$ : geometric mean ratio $(\mathrm{GMR}) ; 90 \%$ confidence interval (CI) $\left.2.79(2.35,3.31)\right]$ while co-administration with diltiazem resulted in a lesser effect [GMR (90\% CI): $2.05(1.82,2.30)$ ]. Co-administration with rifampin led to a marked decrease (88\%) in suvorexant exposure.

Consistent with morning administration and known suvorexant pharmacology, somnolence was the most frequently reported $\mathrm{AE}$.

Conclusions-These results are consistent with expectations that strong CYP3A inhibitors and inducers exert marked effects on suvorexant pharmacokinetics. In the context of a limited sample size, single suvorexant doses were generally well tolerated in healthy subjects when coadministered with/without a CYP3A inhibitor/inducer.

\section{Introduction}

Orexin antagonism has been extensively studied in the past decade as an alternative mechanism for the treatment of insomnia [1, 2]. Orexin A (OX-A) and orexin B (OX-B) are the key signaling neuropeptides in the orexinergic signaling pathway, which plays a central role in regulation of the transition between wake and sleep [3-5]. These neuropeptides exert their wake-promoting effects by activating their cognate orexin receptors $\left(\mathrm{OX}_{1} \mathrm{R}\right.$ and $\left.\mathrm{OX}_{2} \mathrm{R}\right)$ in the posterolateral hypothalamus and brain stem nuclei $[2,6,7]$. Suvorexant is an orally active, first-in-class orexin receptor antagonist indicated in the USA and other regions for the treatment of insomnia, characterized by difficulties with sleep onset and/or sleep maintenance $[8,9]$. Data from clinical trials have demonstrated the efficacy of suvorexant in promoting sleep onset and maintenance with minimal residual next-day effects [10-16]. As the target patient population may include those with comorbidities and elderly patients, polypharmacy may be common in the treatment of insomnia and, as such, evaluation of the potential for drug-drug interactions (DDIs) is critical to inform both prescribing information and clinical practice.

Recent draft guidance from the US Food and Drug Administration (FDA) recommends that DDI management strategies should be developed when a clinically significant DDI for a drug is identified. These strategies may include contraindicating concomitant drug use, avoidance of concomitant drug use, temporary discontinuation of an interacting drug, dosage modifications of the new and concomitant drug, and specific monitoring strategies [17]. The overall objective of these DDI assessments is to provide an understanding of the mechanisms, magnitude, and potential consequences of any given DDI in order to optimize clinical response [17]. Cytochrome P450 3A (CYP3A) inhibition can increase exposure to gamma-aminobutyric acid $(\mathrm{GABA})_{\mathrm{A}}$-receptor hypnotics that are CYP3A substrates (e.g. zolpidem, eszopiclone) $[18,19]$. It therefore follows that co-administration of CYP3A inhibitors and inducers with suvorexant, a CYP3A substrate [8], may alter suvorexant plasma concentrations, and subsequently, its therapeutic effect and safety profile. In vitro and in vivo characterization of suvorexant metabolism and disposition following single oral dose administration in humans has been reported else-where [20].

Based on the draft 2017 FDA DDI guidance, ketoconazole is designated as a clinical example of a strong CYP3A inhibitor [21] and is known to reduce metabolism of CYP3A 
substrates such as triazolam and alprazolam [22]. Although itraconazole or clarithromycin may reflect the preferred strong CYP3A index inhibitors for clinical investigation, at the time of suvorexant clinical development, ketoconazole was routinely utilized in clinical trials and its designation as a strong CYP3A inhibitor remains. As such, results from DDI trials with ketoconazole may be extrapolated to other examples of strong CYP3A inhibitors such as itraconazole [21]. Diltiazem, a calcium channel antagonist [23], has commonly been regarded as a moderate CYP3A inhibitor, such that the effects of diltiazem on CYP3A substrates may be extrapolated to the setting of co-administration with other moderate CYP3A inhibitors such as erythromycin [21]. Although the preferred categorization of diltiazem continues to evolve, the results reported herein are consistent with its designation and assessment as a moderate CYP3A inhibitor. Conversely, rifampin (rifampicin) is a strong inducer of CYP3A [17, 21], and is known to induce several-fold increases in hepatic and intestinal CYP3A protein expression [24]. Induction of CYP3A expression by rifampin can ultimately lead to $>90 \%$ decreases in plasma concentration of sensitive CYP3A substrates, and has been shown to reduce plasma concentration and effects of several $\mathrm{GABA}_{\mathrm{A}}$-receptor hypnotics (e.g. triazolam, zolpidem) [25, 26].

Two Phase I, open-label, fixed-sequence clinical trials in healthy subjects evaluated the potential for CYP3A-mediated DDIs during the administration of suvorexant. Specifically, the pharmacokinetics of suvorexant were assessed following co-administration with ketoconazole, diltiazem, or rifampin. The safety and tolerability of suvorexant in the presence and absence of these inhibitors/inducers were also evaluated.

\section{Methods}

Two Phase I, open-label, fixed-sequence clinical studies [Merck Sharp \& Dohme Corp., a subsidiary of Merck \& Co., Inc., Kenilworth, NJ, USA, Protocol MK-4305-008 (referred to as "P008") and MK-4305-038 ("P038")] were performed. Study P008 assessed the potential for a DDI arising from co-administration of suvorexant with ketoconazole, and Study P038 assessed co-administration of suvorexant with diltiazem or rifampin.

Study P008 was conducted at ProMedica Clinical Research Center, Inc., MA, USA, and Study P038 at Thomas Jefferson University Clinical Research Unit, PA, USA. Both studies were conducted in accordance with the principles of Good Clinical Practice and were approved by the appropriate institutional review boards and regulatory agencies. All subjects provided informed consent.

\subsection{Study Populations and Procedures}

Subjects in Study P008 were healthy men aged 18-45 years, with a body mass index (BMI) of $\$ 31 \mathrm{~kg} / \mathrm{m}^{2}$ at pre-study screening. Study P038 subjects were healthy men and healthy non-pregnant women aged $18-50$ years, with a BMI of $\geq 20$ to $\$ 0 \mathrm{~kg} / \mathrm{m}^{2}$ at pre-study screening. In both trials, subjects were non-smokers and in good overall mental and physical health based on vital signs, medical history, physical examination, laboratory safety tests, and had no abnormality on electrocardiograms (ECG) performed at screening. Those with a history of persistent difficulties in initiating or maintaining sleep for $\geq 3$ months and those with obstructive sleep apnea, restless legs syndrome, narcolepsy, rapid eye movement, 
behavioral disorders (Study P038 only), or difficulty sleeping with sleep aids (Study P038 only) were excluded. In addition, subjects who could not refrain from prescription and/or non-prescription drugs and herbal remedies, and those who were frequent users of sedative hypnotics, including benzodiazepines, 'soft' GABAnergic agents, barbiturates, and/or other pharmaceutical sleep agents, were excluded. Subjects who had crossed three or more time zones in the 2 weeks prior to study initiation were also excluded from Study P008 only.

\subsection{Treatments}

Both studies consisted of two periods: in Period 1, subjects received a single oral dose of suvorexant (Merck Sharp \& Dohme Corp., a subsidiary of Merck \& Co., Inc., Whitehouse Station, NJ, USA) only; in Period 2, they received a single oral dose of suvorexant coadministered with a CYP3A inhibitor or inducer (Fig. 1). All dosing occurred once daily in the morning. Selected CYP3A inhibitor and inducer doses were based on those known to be clinically relevant and/or able to maximize the inhibitory or induction potential described for CYP3A substrates [27-29].

Suvorexant doses were guided in part by pharmacokinetic data from a rising single-dose clinical study in healthy males [30]. Assuming a maximum 10-fold increase in suvorexant area under the concentration-time curve from time of administration to infinity $\left(\mathrm{AUC}_{0-\infty}\right)$ and maximum observed concentration $\left(C_{\max }\right)$ as a result of co-administration of ketoconazole, the suvorexant 4-mg dose selected for Study P008 would still have been associated with a lower exposure and similar $C_{\max }$ compared with the well-tolerated 120-mg dose in the rising single-dose study. This approach was considered conservative, given that only a moderate increase in suvorexant exposure was expected in the presence of ketoconazole, based on published data for midazolam and triazolam [31, 32], which are extensively metabolized by CYP3A [33]. Two days of ketoconazole administration is sufficient to achieve maximal CYP3A inhibition prior to suvorexant dosing; however, due to the approximately 240 -h pharmacokinetic sampling scheme required for suvorexant characterization, and the need to ensure continued ketoconazole-mediated CYP3A inhibition throughout the sample duration, ketoconazole was administered for an additional 9 days.

Co-administration of diltiazem was anticipated to have a lesser impact. Assuming a conservative maximum 5-fold increase in suvorexant $\mathrm{AUC}_{0-\infty}$ and $C_{\max }$ as a result of coadministration of diltiazem, the suvorexant 20-mg dose selected for Study P038 would have been associated with lower exposure $\left(\mathrm{AUC}_{0-\infty}\right.$ and $C_{\mathrm{max}}$ ), compared with the generally well-tolerated 240-mg dose in the rising single-dose study. Substantial CYP3A inhibition can be achieved with one day of diltiazem administration, but to ensure continued diltiazemmediated CYP3A inhibition during the 120-h suvorexant pharmacokinetic sampling scheme, diltiazem was co-administered with suvorexant on Day 2 and for 4 subsequent days following suvorexant administration.

The selection of suvorexant $40 \mathrm{mg}$ was largely informed by the maximum clinical dose that was investigated in parallel during Phase III clinical development, and owing to the pharmacokinetic properties across the clinically relevant dose range $(10-40 \mathrm{mg})$, any effect of rifampin on suvorexant $40 \mathrm{mg}$ could also support similar conclusions at the lower doses including those currently approved of 10-20 mg. In the current study, rifampin was 
administered for 13 days prior to administration of suvorexant to allow near-maximal levels of induction to be achieved, and then co-administered with a single suvorexant dose. Rifampin was further administered for an additional 3 days throughout the $96 \mathrm{~h}$ of suvorexant pharmacokinetic sampling to ensure continued CYP3A induction during this period for a total of 17 days.

\subsection{Pharmacokinetic Evaluations}

Venous blood samples were collected at prespecified time points for suvorexant plasma concentration analysis in both studies (Fig. 1; Table 1). Suvorexant pharmacokinetic parameters were evaluated and included: $\mathrm{AUC}_{0-\infty}, C_{\max }$, apparent terminal elimination halflife ( $\left.t_{1 / 2}\right)$, and time to reach $C_{\max }\left(t_{\max }\right)$. These parameters were assessed following administration of suvorexant alone, and following co-administration with ketoconazole, diltiazem, or rifampin.

\subsection{Safety Assessments}

Subjects were queried daily throughout the trials for the occurrence of adverse events (AEs). All AEs were evaluated with respect to seriousness, severity [mild (awareness of sign or symptom but easily tolerated), moderate (discomfort enough to cause interference with usual activity), severe (incapacitating with inability to work or do usual activity)], relation to trial drug, and action taken in response to their emergence. AEs considered events of clinical interest (ECIs) included: cataplexy, sleep paralysis (including sleep-onset paralysis), hypnagogic or hypnopompic hallucinations, suicidal ideation and/or behaviors, complex sleep-related behaviors, falls, and events associated with potential for abuse. Physical examinations were conducted at screening, pre-dose, and $24 \mathrm{~h}$ post-dose (ketoconazole only), and at the post-trial visit (14 days following the last dose of inhibitor/inducer). Vital signs measurements, in addition to ECG and laboratory safety analyses (hematology, serum chemistry, and urinalysis) were conducted at screening, pre-dose, and various time points between 1 to 8 and $24 \mathrm{~h}$, and Day 3 (for Study P038 only) post-dose, and at the post-trial visit (14 days following the last dose of inhibitor/inducer).

\subsection{Sample Analysis, Data Analysis, and Statistics}

Plasma concentrations of suvorexant (molecular weight: $450.932 \mathrm{~g} / \mathrm{mol}$ ) were determined by liquid-liquid extraction followed by quantification using reversed-phase high-performance liquid chromatography-mass spectrometry [34]. The lower limit of quantification of the assay and its linear calibration range were $1 \mathrm{ng} / \mathrm{mL}$ and $1-1000 \mathrm{ng} / \mathrm{mL}$, respectively. Suvorexant plasma concentrations, and their corresponding sampling times relative to the dosing time, were used to derive the pharmacokinetic parameters in each subject using the WinNonlin software (version 5.1.1/5.2.1; Pharsight Corporation, Mountain View, CA, USA). The apparent terminal rate constant $(\lambda)$ was estimated by regression of the terminal loglinear portion of the plasma concentration-time profile. AUC from time of administration to the last time point of detectable plasma concentration $\left(\mathrm{AUC}_{0-\text { last }}\right)$ was calculated using the linear trapezoidal method for ascending concentrations and the log trapezoidal method for descending concentrations up to the last detectable plasma concentration. $\mathrm{AUC}_{0-\infty}$ was estimated as the sum of $\mathrm{AUC}_{0 \text {-last }}$ and the extrapolated area given by the quotient of the last detectable concentration and $\lambda . C_{\max }$ was obtained by inspection of the plasma 
concentration data. $t_{\max }$ was obtained by inspection of the plasma concentration data and, finally, the $t / 2$ was calculated as the quotient of $\ln (2)$ and $\lambda$.

Individual $\mathrm{AUC}_{0-\infty}$ values were $\ln$-transformed and evaluated with a mixed-effect analysis of variance (ANOVA) model, with treatment as a fixed effect and subject as a random effect. A two-sided $90 \%$ confidence interval (CI) for the true mean difference (suvorexant with CYP3A inhibitor or inducer-suvorexant alone) in ln-AUC was calculated using the mean square error from the ANOVA and referencing a t-distribution. These confidence limits were exponentiated to obtain a $\mathrm{CI}$ for the true geometric mean ratio (GMR) for $\mathrm{AUC}_{0-\infty}$ (suvorexant with CYP3A inhibitor or inducer/suvorexant alone). Suvorexant $C_{\max }$ was analyzed in a similar manner. No-effect boundaries of 0.5-2.0 were selected to inform sample size of the current studies and preliminary clinical development and trial conduct criteria. The totality of evidence, including clinical efficacy and safety data, and other approaches provide conclusive recommendations and interpretation of clinical relevance for any drug interactions and dosing guidelines.

AEs were tabulated for administration of suvorexant alone and in the presence of ketoconazole, diltiazem, or rifampin by frequency of occurrence. For vital signs, laboratory safety tests, and ECG monitoring, summary statistics of mean and standard error were generated for values and changes from baseline by part, treatment, and time point. Selected non-serious AEs (ECIs) were recorded and consisted of cataplexy, hypnagogic or hypnopompic hallucinations, sleep paralysis or sleep-onset paralysis, suicidal ideation and/or behaviors, complex sleep-related behaviors, falls, and selected events associated with potential for abuse.

Across both studies, sample sizes were selected based on study objectives and the pharmacokinetic variability of suvorexant established at the time of study conduct. Assuming a true GMR of 1.0, each of the sample sizes were expected to achieve a $\geq 80 \%$ probability that the $90 \%$ CI would fall between 0.5 and 2.0.

\section{Results}

\subsection{Study Populations}

Study P008 was conducted from November to December 2008, and Study P038 was conducted from August to October 2010, with treatment periods of approximately 4 and 8 weeks, respectively. Ten male subjects enrolled in and completed Study P008 (Table 2). A total of 30 subjects were enrolled in Study P038: 20 in the diltiazem sub-study, and 10 in the rifampin sub-study (Table 2). Seventeen and 10 subjects completed the diltiazem and rifampin sub-studies, respectively. In the diltiazem sub-study, one subject discontinued at the post-study visit having completed Periods 1 and 2 .

All subjects were included in the pharmacokinetic analyses, with complete data available for all 10 (100\%) subjects in Study P008, 18/20 [90\% (including the subject who discontinued at the post-study visit)] in the P038 diltiazem sub-study, and all 10 (100\%) in the P038 rifampin sub-study. Two subjects in Study P038 (both from the diltiazem sub-study) did not 
have a complete pharmacokinetic data set, as only data for Period 1 were available. Across both studies, all subjects $(N=40)$ were included in the safety evaluation.

\subsection{Pharmacokinetic Evaluations}

Arithmetic mean plasma concentration-time profiles for suvorexant alone and following coadministration with ketoconazole (400 mg), diltiazem (240 mg), or rifampin (600 mg) are provided in Fig. 2a-c, and the corresponding pharmacokinetic parameters are described in Table 3.

GMRs and their associated 90\% CIs for the comparison of suvorexant in the presence and absence of inhibitors or an inducer of CYP3A are presented in Fig. 3. Co-administration of ketoconazole resulted in a moderate increase in suvorexant exposure $\left(\mathrm{AUC}_{0-\infty}\right)$ while coadministration of diltiazem resulted in a lesser effect. In contrast to exposure, marked differences in suvorexant $C_{\max }$ were not observed following co-administration with strong or moderate CYP3A inhibitors. In contrast to co-administration of ketoconazole or diltiazem, co-administration of rifampin resulted in a decrease in suvorexant exposure $\left(\mathrm{AUC}_{0-\infty}, 88 \%\right)$ and suvorexant $C_{\max }(64 \%)$.

Compared with suvorexant administered alone ( $t / 1 / 2$ range $11.2-12.9 \mathrm{~h})$, suvorexant $t / 2$ was prolonged when co-administered with ketoconazole (19.4 h) or diltiazem (16.1 h; Table 3) and decreased with rifampin co-administration $(7.70 \mathrm{~h}$; Table 3$)$.

\subsection{Safety Assessments}

The incidence of AEs is summarized in Table 4 for both trials. In Study P008, six (60\%) subjects reported at least one AE with suvorexant administered alone and seven (70\%) subjects reported at least one $\mathrm{AE}$ when suvorexant was co-administered with ketoconazole. In the P038 diltiazem sub-study, 20 (100\%) subjects reported at least one AE with suvorexant administered alone and $18(90 \%)$ with suvorexant co-administered with diltiazem (Table 4). All 10 (100\%) subjects in the P038 rifampin sub-study reported at least one $\mathrm{AE}$, with both suvorexant administered alone and when co-administered with rifampin (Table 4). Somnolence was the most frequently reported AE across all treatments for both studies, consistent with the reported pharmacology of suvorexant [8].

All AEs were mild in intensity, except one moderate AE of headache reported in the P038 diltiazem sub-study, which resolved without intervention. All AEs in Study P008, and the majority of AEs in Study P038, were considered probably or possibly related to study treatment by the investigator. There were no serious AEs or deaths in either trial.

In Study P008, no AEs were considered to be ECIs. In Study P038, five AEs were considered ECIs (diltiazem sub-study, $n=1$; rifampin sub-study, $n=4$ ). These events were: euphoric mood $(n=1)$ with suvorexant $20 \mathrm{mg}$ plus diltiazem, and euphoric mood $(n=1)$, sleep paralysis $(n=1)$, hypnagogic hallucination $(n=1)$, and hypnopompic hallucination $(n$ $=1$ ) with suvorexant $40 \mathrm{mg}$ alone in the rifampin sub-study. These events were assessed as possibly related to study treatment and short-lived in duration, ranging from $1 \mathrm{~min}$ [hypnopompic hallucination (suvorexant $40 \mathrm{mg}$ in the rifampin sub-study)] to $1 \mathrm{~h}$ [euphoric mood (suvorexant $20 \mathrm{mg}$ plus diltiazem)]. 
Across both studies, no clinically meaningful changes in vital signs, laboratory safety tests, physical examination, or ECG monitoring were observed. Although some laboratory values fell outside the normal range, these variations were considered minor and, therefore, not clinically significant by the investigator and clinical monitor.

\section{Discussion}

Clinical pharmacokinetic DDI studies in healthy subjects were conducted with ketoconazole (strong CYP3A inhibitor), diltiazem (moderate CYP3A inhibitor), and rifampin (strong CYP3A inducer) to evaluate the potential for suvorexant to be subject to drug interactions mediated via inhibition or induction of CYP3A. Overall, CYP3A inhibition increased suvorexant exposure, while CYP3A induction decreased suvorexant exposure. Both studies used morning administration of suvorexant, and consistent with the known pharmacology of suvorexant, somnolence was the most widely reported AE [8].

Consistent with expectations of a CYP3A substrate, administration of suvorexant with strong or moderate CYP3A inhibitors resulted in increases in exposure $\left(\mathrm{AUC}_{0-\infty}\right)$. When suvorexant was co-administered with the strong CYP3A inhibitor ketoconazole or the moderate CYP3A inhibitor diltiazem, there were increases in suvorexant plasma concentrations compared with suvorexant alone (2.79- and 2.05-fold increases in $\mathrm{AUC}_{0-\infty}$, respectively), with a prolongation in $t_{1 / 2}$ (ketoconazole study, an increase of $8.2 \mathrm{~h}$; diltiazem sub-study, an increase of $3.7 \mathrm{~h}$ ). The observed increase in $t_{1 / 2}$ with minimal increase in $C_{\max }$ suggests that the observed increase in suvorexant $\mathrm{AUC}_{0-\infty}$ following co-administration with ketoconazole may be due to an effect on systemic clearance, rather than on pre-systemic metabolism. While suvorexant is primarily cleared by CYP3A metabolism [8], the $<5$-fold increase in $\mathrm{AUC}_{0-\infty}$ following co-administration with ketoconazole was considered moderate and is likely due to the low clearance of suvorexant. When single doses of suvorexant $40 \mathrm{mg}$ were co-administered with multiple doses of the strong CYP3A inducer rifampin, suvorexant exposure was reduced compared with when suvorexant was administered alone (approximately $88 \%$ reduction in suvorexant $\mathrm{AUC}_{0-\infty}$ and $64 \%$ reduction in suvorexant $C_{\mathrm{max}}$ ). The larger impact of rifampin administration on suvorexant $\mathrm{AUC}_{0-\infty}$ compared with $C_{\max }$ indicates that the effects of this drug are mediated largely by changes in systemic clearance-related mechanisms. Consistent with previously reported pharmacokinetic parameters [35], suvorexant was readily absorbed, with a median $t_{\max }$ of 1$2 \mathrm{~h}$ in both trials summarized herein.

Although doses of suvorexant $20 \mathrm{mg}$ were not specifically evaluated in the present trials, since the relative bioavailability between 10 and $20 \mathrm{mg}$ tablets is equivalent, and suvorexant displays linear (time- and dose-independent) systemic pharmacokinetics (data on file), the anticipated suvorexant exposures in the presence of CYP3A inhibitors or inducers can be expected to be similar to those reported in the trials summarized herein. Further, given the collective pharmacokinetic data indicating the absence of a significant difference in elderly versus non-elderly healthy subjects and patients [8], the same assumptions may be applicable to elderly and non-elderly populations [12]. 
Notably, the observed increase in suvorexant exposure resulting from reduced CYP3Amediated metabolism may be expected to lead to increased therapeutic effect and potential side effects, such as increases in next-day residual effects. However, in a randomized, double-blind, placebocontrolled, 4-period crossover polysomnography study in healthy subjects, no clinically meaningful effect on psycho-motor performance was observed following single doses of suvorexant 10 and $50 \mathrm{mg}$, whereas a statistically significant prolongation in reaction time (assessed using choice reaction time and simple reaction time) was observed with the $100 \mathrm{mg}$ dose [14]. Suvorexant is approved at doses between 10 and $20 \mathrm{mg}$, and the need for dose adjustment or other clinical considerations in the setting of coadministration with strong or moderate CYP3A inhibitors is dependent on the approved dose in a particular geographical location. Local prescribing information should, therefore, be consulted for guidance. In contrast to co-administration with a CYP3A inhibitor, coadministration with a strong CYP3A inducer is likely to reduce suvorexant exposure and may, therefore, reduce efficacy [8]. In subsequent Phase II and III trials of the suvorexant development program, the concomitant use of mild-to-moderate CYP3A inhibitors (e.g. verapamil and diltiazem) and inducers (e.g. glucocorticoids) was permitted (data on file). Although only a limited number of subjects received these inhibitors $(n=15)$ and inducers $(n=25)$, an associated, significant effect on suvorexant exposure was not observed (data on file). This observation is consistent with expectations that co-administration of suvorexant with these mild-to-moderate inhibitors and inducers would exert much smaller effect sizes on suvorexant exposure than the strong inhibitors and inducers evaluated during Phase I development.

Consistent with morning administration and the known pharmacology of suvorexant [8], somnolence was the most frequently reported $\mathrm{AE}$, with a greater incidence at higher doses of suvorexant.

Five AEs were considered ECIs and were reported following administration of suvorexant $40 \mathrm{mg}$ alone or suvorexant $20 \mathrm{mg}$ plus diltiazem in Study P038, including euphoric mood, hypnagogic and hypnopompic hallucinations, and sleep paralysis. Such sleep-related hallucinations have been previously reported in trials of zolpidem and zaleplon [18, 36].

The effects of CYP3A inhibition and induction are generalizable from single dose to chronic use; however, owing to the small sample size of the trials, the overall clinical response, efficacy, and safety should be informed by risk:benefit assessments.

\section{Conclusions}

CYP3A inhibition and induction altered the pharmacokinetic profile of single-dose suvorexant in healthy subjects. The relative magnitude of the impact was consistent with the effects expected of strong or moderate CYP3A modulators and continues to support the role of CYP3A in the biotransformation of suvorexant. In contrast, weak inhibitors/inducers will be expected to have smaller effect sizes and their impact is unlikely to be clinically relevant. Overall, it is recommended that clinicians refer to local labeling for specific guidance on the prescription of suvorexant in the presence of CYP3A inhibitors or inducers. 


\section{Acknowledgements}

The authors would like to thank Xiaodong Li for serving as a statistician and Hong Sun for serving as a clinical monitor during these trials. Christopher Lines, PhD, and Tamara Cabalu, PhD, of Merck \& Co., Inc., Kenilworth, $\mathrm{NJ}$, USA provided comments and edits on a draft of the manuscript. Medical writing support, under the direction of the authors, was provided by Adele Blair, PhD, of CMC AFFINITY, a division of McCann Health Medical Communications Ltd., Glasgow, UK, in accordance with Good Publication Practice (GPP3) guidelines.

Funding These trials and medical writing support was funded by Merck Sharp \& Dohme Corp., a subsidiary of Merck \& Co., Inc., Kenilworth, NJ, USA.

\section{References}

1. Winrow CJ, Renger JJ. Discovery and development of orexin receptor antagonists as therapeutics for insomnia. Br J Pharmacol. 2014;171(2):283-93. [PubMed: 23731216]

2. Roecker AJ, Coleman PJ. Orexin receptor antagonists: medicinal chemistry and therapeutic potential. Curr Top Med Chem. 2008;8(11):977-87. [PubMed: 18673167]

3. Coleman PJ, Gotter AL, Herring WJ, Winrow CJ, Renger JJ. The discovery of suvorexant, the first orexin receptor drug for insomnia. Annu Rev Pharmacol Toxicol. 2017;57:509-33. [PubMed: 27860547]

4. Gotter AL, Webber AL, Coleman PJ, Renger JJ, Winrow CJ. International Union of Basic and Clinical Pharmacology. LXXXVI. Orexin receptor function, nomenclature and pharmacology. Pharmacol Rev. 2012;64(3):389-420. [PubMed: 22759794]

5. Yoshida Y, Fujiki N, Nakajima T, Ripley B, Matsumura H, Yoneda H, et al. Fluctuation of extracellular hypocretin-1 (orexin A) levels in the rat in relation to the light-dark cycle and sleepwake activities. Eur J Neurosci. 2001;14(7):1075-81. [PubMed: 11683899]

6. Scammell TE, Winrow CJ. Orexin receptors: pharmacology and therapeutic opportunities. Annu Rev Pharmacol Toxicol. 2011;51:243-66. [PubMed: 21034217]

7. Sakurai T, Amemiya A, Ishii M, Matsuzaki I, Chemelli RM, Tanaka H, et al. Orexins and orexin receptors: a family of hypothalamic neuropeptides and $\mathrm{G}$ protein-coupled receptors that regulate feeding behavior. Cell. 1998;92(4):573-85. [PubMed: 9491897]

8. Sharp Merck \& Corp Dohme. Belsomra (suvorexant) Package Insert. 2014 http://www.merck.com/ product/usa/pi_circulars/b/belsomra/belsomra_pi.pdf. Accessed 26 June 2018.

9. Kawabe K, Horiuchi F, Ochi M, Nishimoto K, Ueno SI, Oka Y. Suvorexant for the treatment of insomnia in adolescents. J Child Adolesc Psychopharmacol. 2017;27:792-5. [PubMed: 28520464]

10. Herring WJ, Snyder E, Budd K, Hutzelmann J, Snavely D, Liu K, et al. Orexin receptor antagonism for treatment of insomnia: a randomized clinical trial of suvorexant. Neurology. 2012;79(23):2265-74. [PubMed: 23197752]

11. Herring WJ, Connor KM, Ivgy-May N, Snyder E, Liu K, Snavely DB, et al. Suvorexant in patients with insomnia: results from two 3-month randomized controlled clinical trials. Biol Psychiatry. 2016;79(2):136-48. [PubMed: 25526970]

12. Herring WJ, Connor KM, Snyder E, Snavely DB, Zhang Y, Hutzelmann J, et al. Suvorexant in elderly patients with insomnia: pooled analyses of data from Phase III randomized controlled clinical trials. Am J Geriatr Psychiatry. 2017;25(7):791-802. [PubMed: 28427826]

13. Michelson D, Snyder E, Paradis E, Chengan-Liu M, Snavely DB, Hutzelmann J, et al. Safety and efficacy of suvorexant during 1-year treatment of insomnia with subsequent abrupt treatment discontinuation: a Phase 3 randomised, double-blind, placebo-controlled trial. Lancet Neurol. 2014;13(5):461-71. [PubMed: 24680372]

14. Sun H, Kennedy WP, Wilbraham D, Lewis N, Calder N, Li X, et al. Effects of suvorexant, an orexin receptor antagonist, on sleep parameters as measured by polysomnography in healthy men. Sleep. 2013;36(2):259-67. [PubMed: 23372274]

15. Vermeeren A, Vuurman E, Bautmans A, Li X, Vets E, Lewis N, et al. Suvorexant, a dual orexin receptor antagonist, does not impair next day driving performance in healthy elderly subjects. Sleep. 2012;35(Abstract Supplement):pA226 (Abstract 0670). 
16. Vermeeren A, Vuurman E, Van Oers A, Van Leeuwen C, Jongen S, Bautmans A, et al. Effects of suvorexant, an orexin receptor antagonist on next day driving performance in healthy non-elderly subjects. Neuropsychopharmacology. 2012;38(Suppl 1):S320-1.

17. U.S. Food and Drug Administration. Clinical Drug Interaction Studies — Study Design, Data Analysis, and Clinical Implications Guidance for Industry DRAFT GUIDANCE. 2017 https:// www.fda.gov/downloads/Drugs/GuidanceComplianceRegulatoryInformation/Guidances/ UCM292362.pdf. Accessed 28 June 2018.

18. RxList. Ambien (zolpidem) Package Insert. 2004 https://www.rxlist.com/ambien-drug/patientimages-side-effects.htm. Accessed 29 June 2018.

19. Sunovion Pharmaceuticals Inc. Lunesta [package insert]. 2014 http://www.lunesta.com/ PostedApprovedLabelingText.pdf. Accessed 29 June 2018.

20. Cui D, Cabalu T, Yee KL, Small J, Li X, Liu B, et al. In vitro and in vivo characterisation of the metabolism and disposition of suvorexant in humans. Xenobiotica. 2016;46(10):882-95. [PubMed: 26864332]

21. U.S. Food and Drug Administration. Drug Development and Drug Interactions: Table of Substrates, Inhibitors and Inducers. 2018 https://www.fda.gov/drugs/developmentapprovalprocess/ developmentresources/druginteractionslabeling/ucm093664.htm. Accessed 3 July 2018.

22. Greenblatt DJ, Wright CE, von Moltke LL, Harmatz JS, Ehrenberg BL, Harrel LM, et al. Ketoconazole inhibition of triazolam and alprazolam clearance: differential kinetic and dynamic consequences. Clin Pharmacol Ther. 1998;64(3):237-47. [PubMed: 9757147]

23. Sutton D, Butler AM, Nadin L, Murray M. Role of CYP3A4 in human hepatic diltiazem $N$ demethylation: inhibition of CYP3A4 activity by oxidized diltiazem metabolites. J Pharmacol Exp Ther. 1997;282(1):294-300. [PubMed: 9223567]

24. Niemi M, Backman JT, Fromm MF, Neuvonen PJ, Kivisto KT. Pharmacokinetic interactions with rifampicin : clinical relevance. Clin Pharmacokinet. 2003;42(9):819-50. [PubMed: 12882588]

25. Villikka K, Kivisto KT, Backman JT, Olkkola KT, Neuvonen PJ. Triazolam is ineffective in patients taking rifampin. Clin Pharmacol Ther. 1997;61(1):8-14. [PubMed: 9024169]

26. Villikka K, Kivisto KT, Luurila H, Neuvonen PJ. Rifampin reduces plasma concentrations and effects of zolpidem. Clin Pharmacol Ther. 1997;62(6):629-34. [PubMed: 9433391]

27. Backman JT, Olkkola KT, Neuvonen PJ. Rifampin drastically reduces plasma concentrations and effects of oral midazolam. Clin Pharmacol Ther. 1996;59(1):7-13. [PubMed: 8549036]

28. Stoch SA, Friedman E, Maes A, Yee K, Xu Y, Larson P, et al. Effect of different durations of ketoconazole dosing on the single-dose pharmacokinetics of midazolam: shortening the paradigm. J Clin Pharmacol. 2009;49(4):398-406. [PubMed: 19246722]

29. Friedman EJ, Fraser IP, Wang Y-H, Bergman AJ, Li C-C, Larson PJ, et al. Effect of different durations and formulations of diltiazem on the single-dose pharmacokinetics of midazolam: how long do we go? J Clin Pharmacol. 2011;51(11):1561-70. [PubMed: 21209240]

30. Sun H, Kennedy WD, Lewis N, Laethem T, Tee K, Li X, et al. The single dose pharmacokinetic (PK) and pharmacodynamic (PD) profiles of suvorexant (MK-4305), a dual orexin receptor antagonist, in healthy male subjects. Sleep Biol Rhythms. 2011;9(4):332 (Abstract).

31. Olkkola KT, Backman JT, Neuvonen PJ. Midazolam should be avoided in patients receiving the systemic antimycotics ketoconazole or itraconazole. Clin Pharmacol Ther. 1994;55(5):481-5. [PubMed: 8181191]

32. Varhe A, Olkkola KT, Neuvonen PJ. Oral triazolam is potentially hazardous to patients receiving systemic antimycotics ketoconazole or itraconazole. Clin Pharmacol Ther. 1994;56(6 Pt 1):601-7. [PubMed: 7995001]

33. Kronbach T, Mathys D, Umeno M, Gonzalez FJ, Meyer UA. Oxidation of midazolam and triazolam by human liver cytochrom P450IIIA4. Mol Pharmacol. 1989;36:89-96. [PubMed: 2787473]

34. Breidinger SA, Simpson RC, Mangin E, Woolf EJ. Determination of suvorexant in human plasma using 96-well liquid-liquid extraction and HPLC with tandem mass spectrometric detection. J Chromatogr B Analyt Technol Biomed Life Sci. 2015;1002:254-9. 
35. Yee KL, McCrea J, Panebianco D, Liu W, Lewis N, Cabalu T, et al. Safety, tolerability, and pharmacokinetics of suvorexant: a randomized rising-dose trial in healthy men. Clin Drug Invest. 2018;38(7):631-8.

36. Drugs.com. Sonata (zaleplon) Package Insert. 2004 https://www.drugs.com/sonata.html. Accessed 3 July 2018. 


\section{Key Points}

- The pharmacokinetic interactions of inhibitors (ketoconazole or diltiazem) or an inducer (rifampin) of CYP3A on suvorexant are summarized.

- Co-administration of ketoconazole or diltiazem with suvorexant resulted in increases in suvorexant $\mathrm{AUC}_{0-\infty}$, and co-administration of rifampin with suvorexant resulted in a decrease in suvorexant $\mathrm{AUC}_{0-\infty}$.

- $\quad$ Single suvorexant doses were generally well tolerated in healthy subjects, both alone and when co-administered with a CYP3A inhibitor or inducer. 
(a)

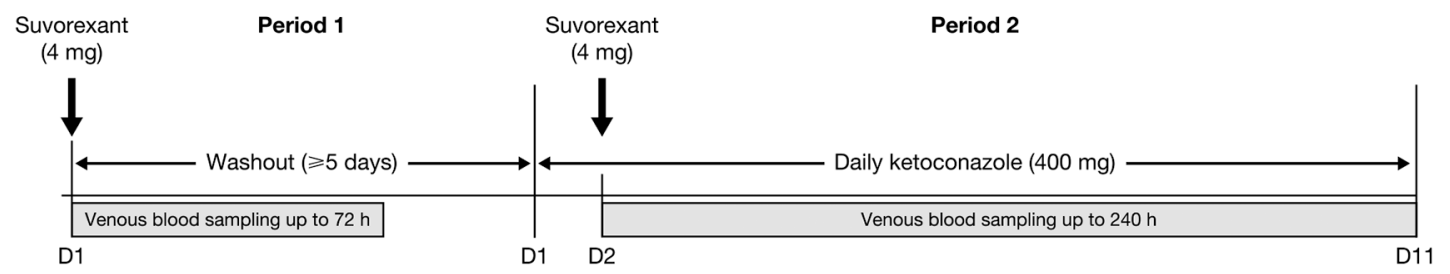

(b)

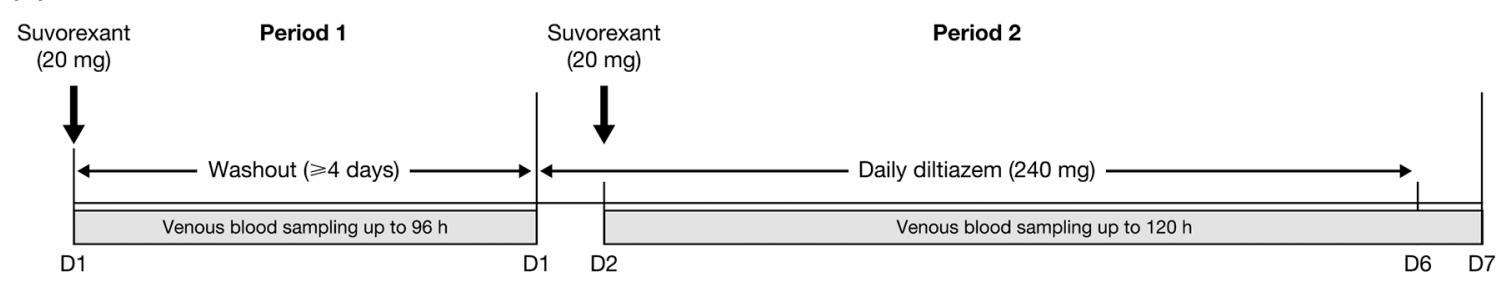

(c)

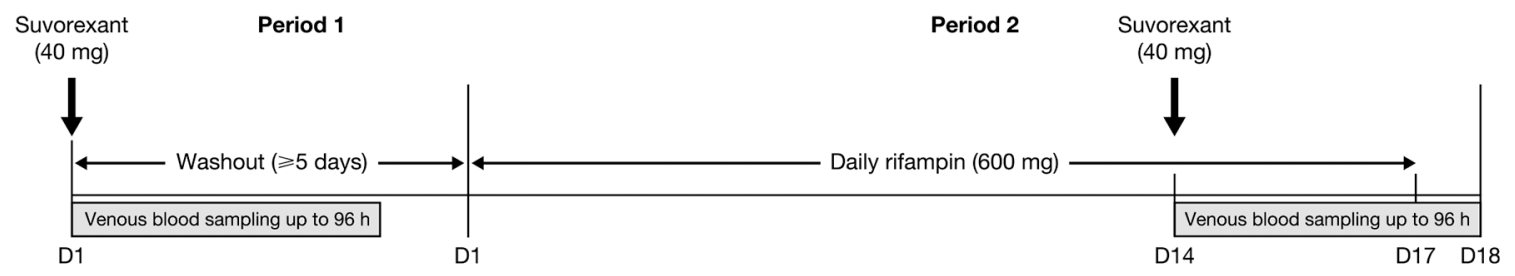

Fig. 1.

Study design for a Study P008, b Study P038 diltiazem sub-study, and c Study P038

rifampin sub-study. $D$ day, $h$ hours 


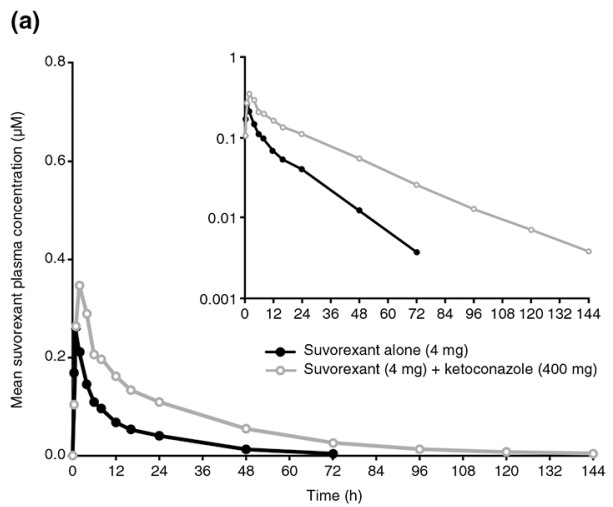

(b)

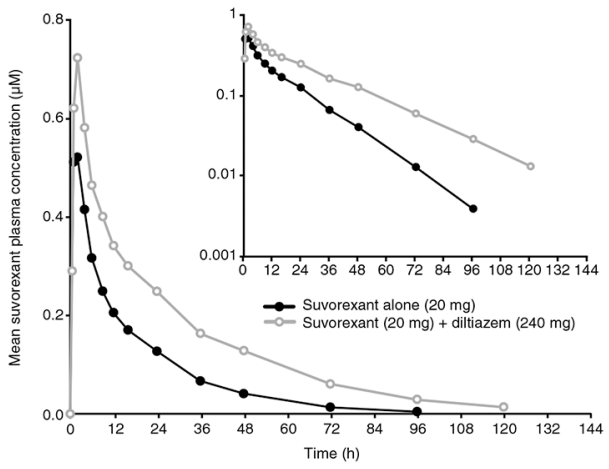

(c)

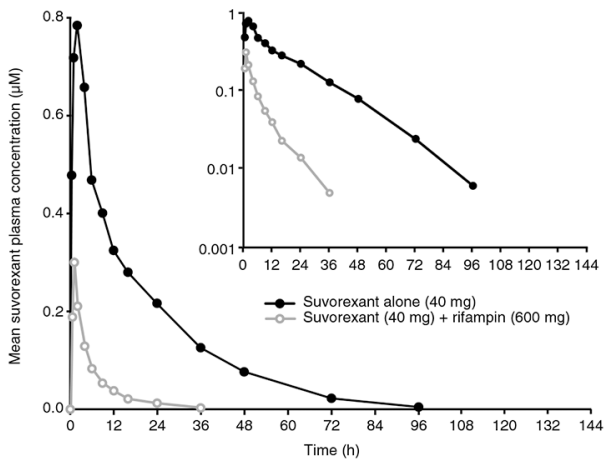

Fig. 2.

Arithmetic mean plasma concentration-time profile of suvorexant following administration of suvorexant alone or co-administered with a the strong CYP3A inhibitor ketoconazole $(N$ $=10$, Study P008), $\mathbf{b}$ the moderate CYP3A inhibitor diltiazem (suvorexant alone, $N=20$; suvorexant plus diltiazem, $N=18$; Study P038), or c the strong CYP3A inducer rifampin $(N$ $=10$, Study P038), in healthy subjects. Inset: semilog scale. $C Y P$ cytochrome P450, $h$ hours 
Interacting drug

Strong CYP3A inhibitor:

Ketoconazole $400 \mathrm{mg}$

Moderate CYP3A inhibitor:

Diltiazem $240 \mathrm{mg}$

\section{Strong CYP3A inducer:}

Rifampin $600 \mathrm{mg}$

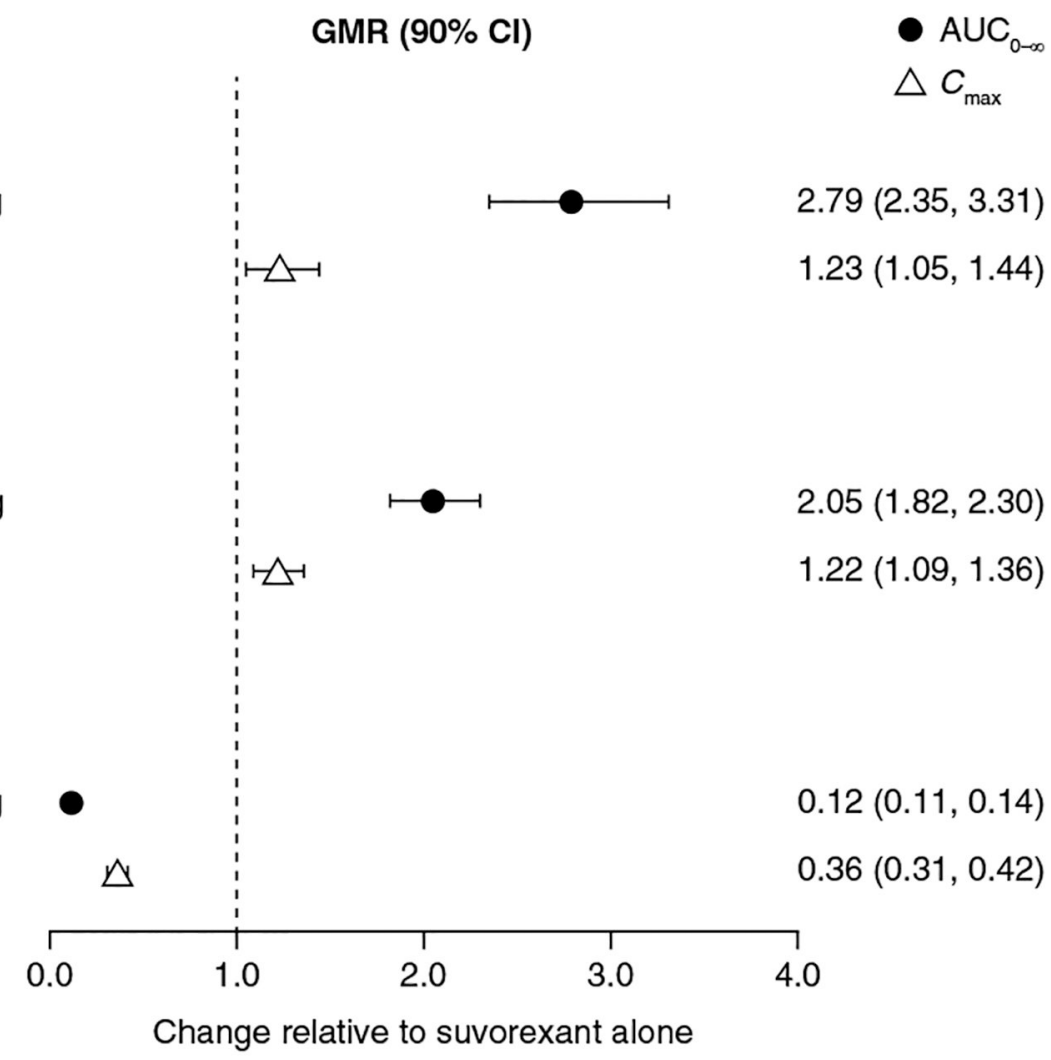

Fig. 3.

GMRs $(90 \% \mathrm{CI})$ for suvorexant co-administered with the strong CYP3A inhibitor ketoconazole, the moderate CYP3A inhibitor diltiazem, or the strong CYP3A inducer rifampin, versus suvorexant alone, for suvorexant $\mathrm{AUC}_{0-\infty}$ (top) and $C_{\max }$ (bottom). $A U C_{0-\infty}$ area under the concentration-time curve from time of administration to infinity, $C I$ confidence interval, $C_{\max }$ maximum observed concentration, $C Y P$ cytochrome $\mathrm{P} 450, G M R$ least-squares geometric mean ratio 


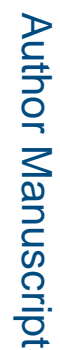

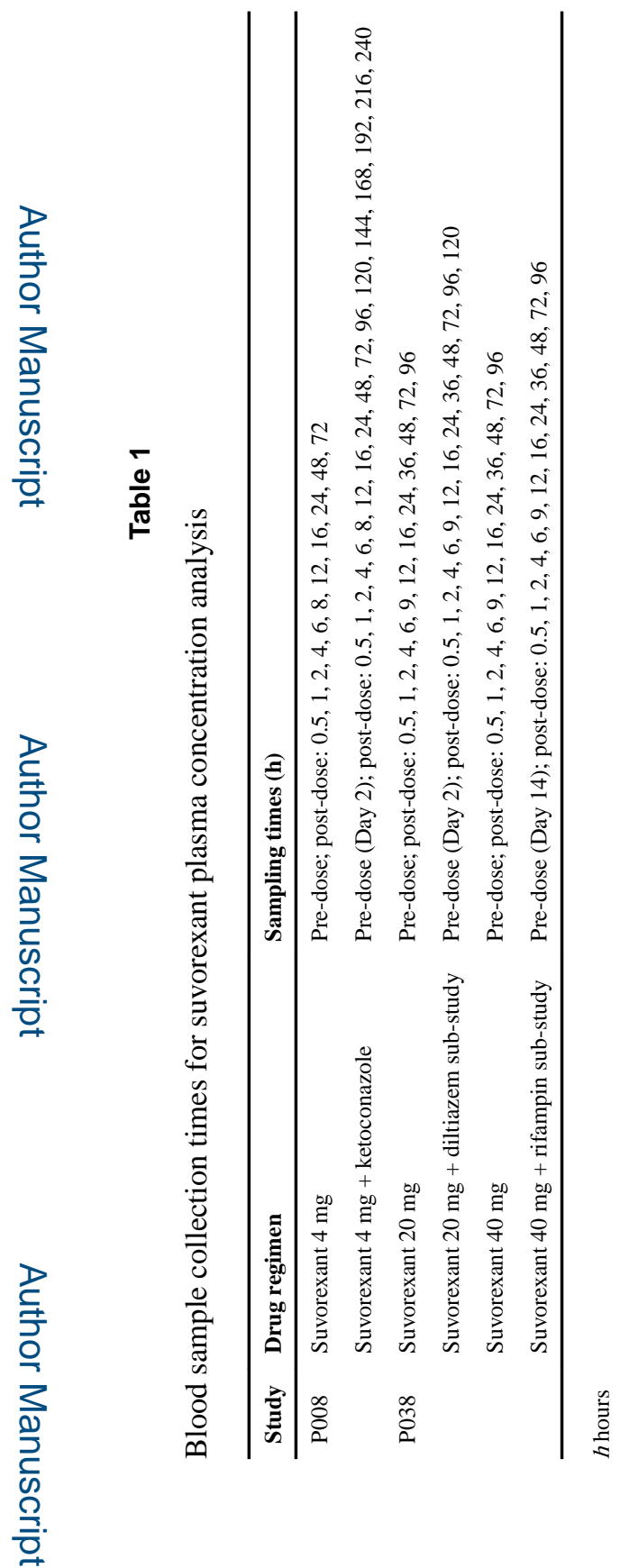

Clin Drug Investig. Author manuscript; available in PMC 2020 May 01. 


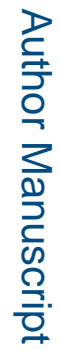

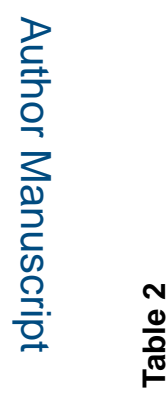

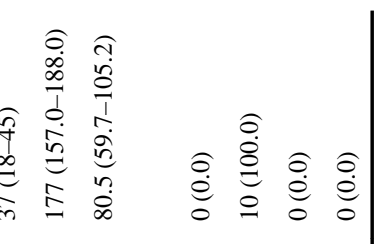

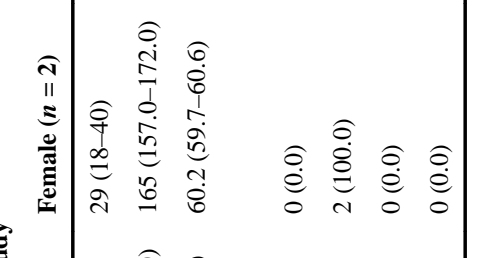

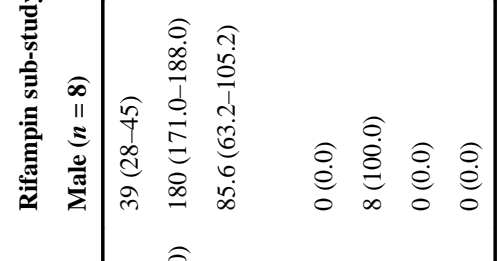

$\widehat{\approx} \stackrel{\partial}{\sigma} \curvearrowright$

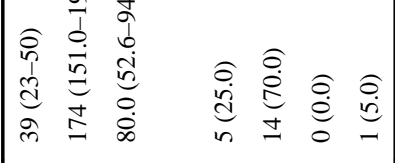

$\infty$

- $\infty$ in -0

疍

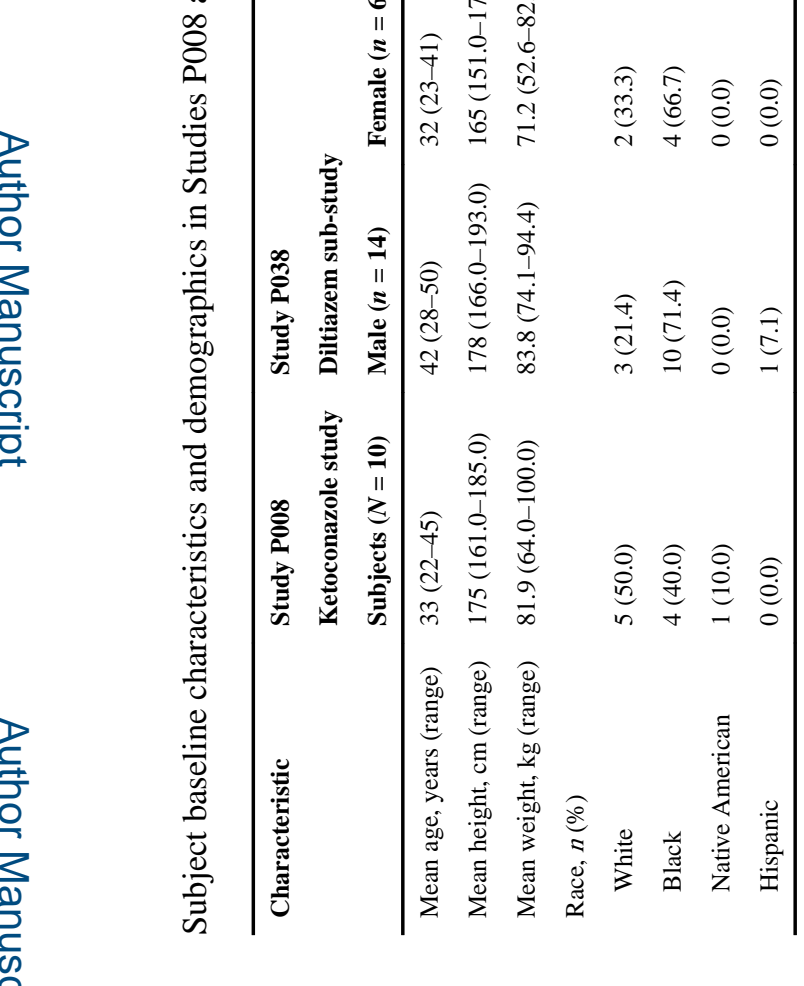

일

음 
Wrishko et al.

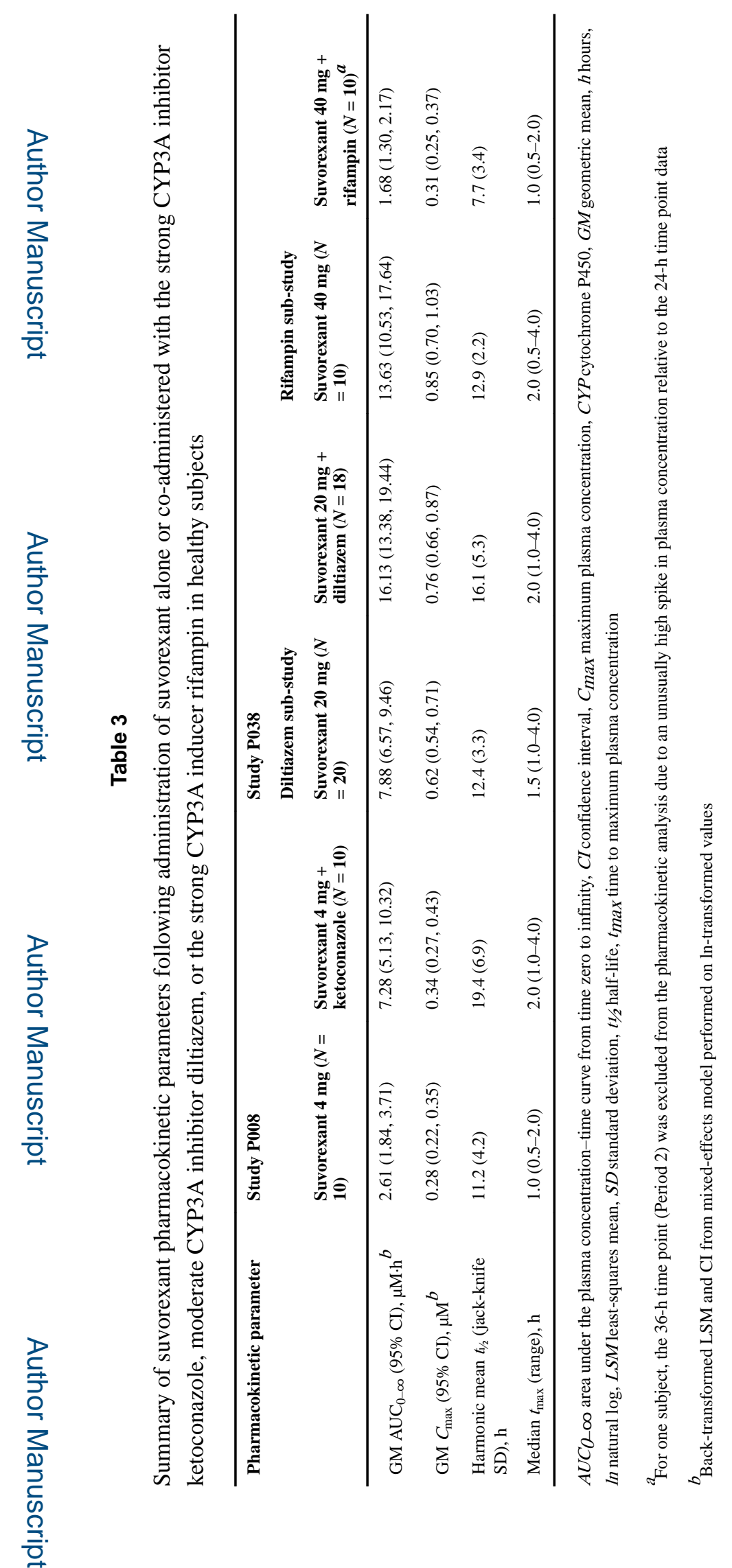

Clin Drug Investig. Author manuscript; available in PMC 2020 May 01. 


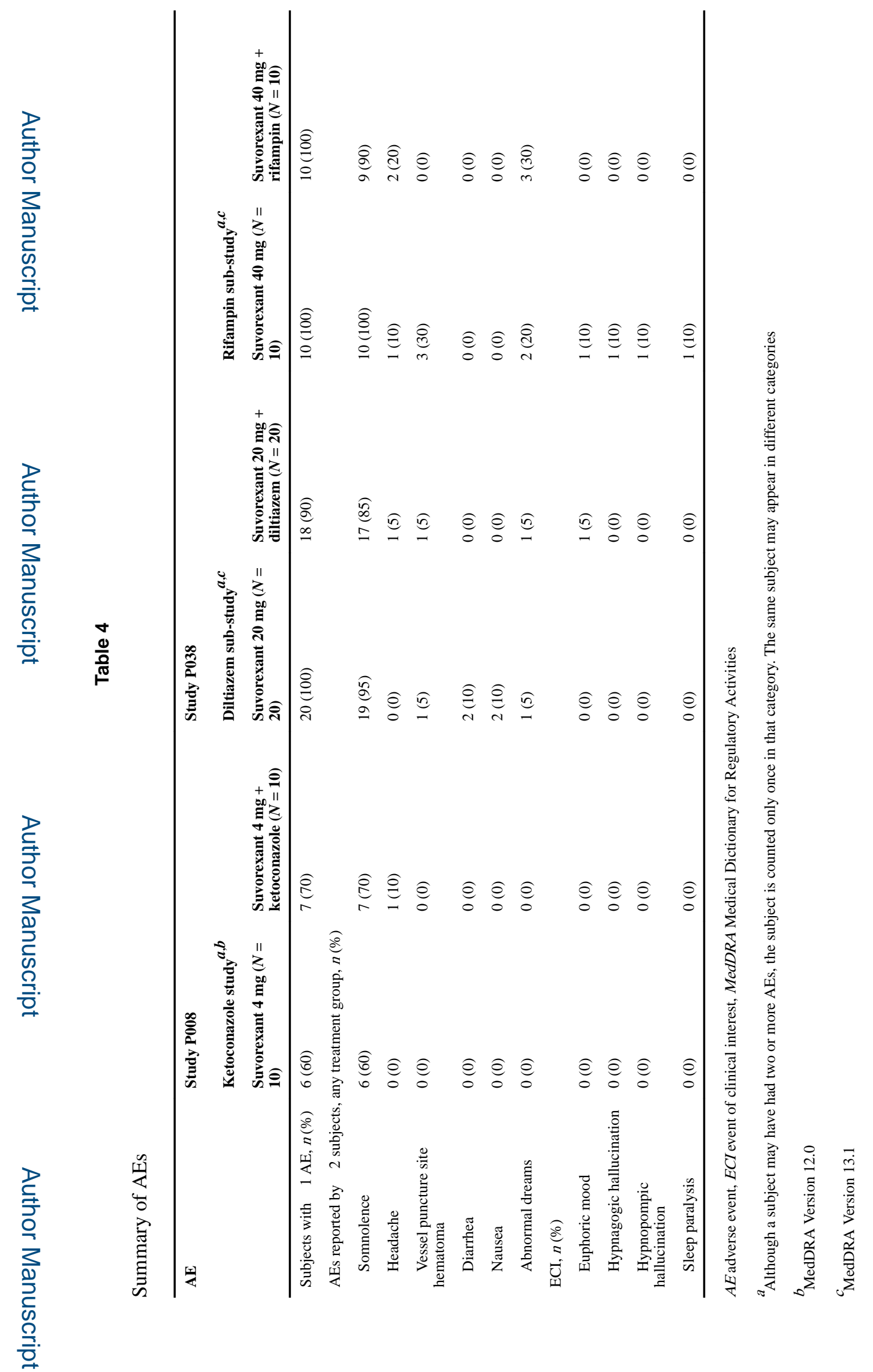

Clin Drug Investig. Author manuscript; available in PMC 2020 May 01. 